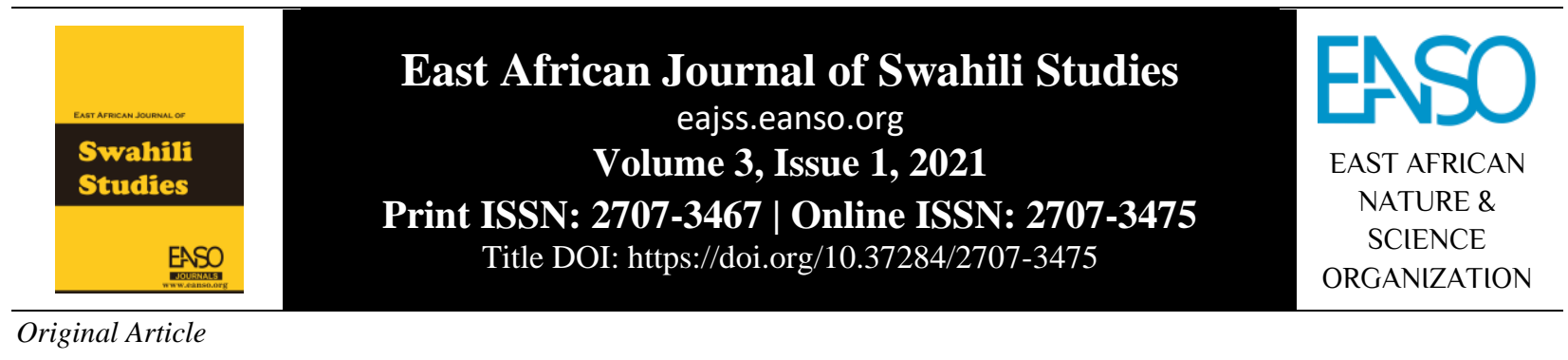

\title{
Si Tendi zothe za Kabla ya Karne ya Ishirini ni Utambaji wa Kingano Nyengine ni Ufunzaji: Kiyelelezo cha Uțendi wa Siri li Asrari
}

\author{
Mohamed Karamal, Rocha Chimerah ${ }^{2}$ \& Kineene wa Mutiso ${ }^{3}$ \\ ${ }^{1}$ Chuo Kikuu cha Kabianga, S.L.P 2030 - 20200, Nairobi, Kenya. \\ * ORCID ID: https://orcid.org/0000-0003-2341-9966; Barua pepe ya mawasiliano: mkarama@kabianga.ac.ke
}

DOI ya Nakala: https://doi.org/10.37284/eajss.3.1.349

\section{Tarehe ya Uchapishaji: IKISIRI}

26 Juni 2021 Fikra iliyomo katika mawanda ya fasihi ya Kiswahili ni kuwa utendi ni maelezo marefu ya mapisi ya jamii kiushairi. Makala haya yanataka kuonesha kwamba Istilahi Muhimu: si tendi zothe zenye madhumuni haya. Kupitiya Utendi wa Siri li Asrari tutaonesha kwamba $\mathrm{mt}^{\mathrm{h}}$ unzi Mwanalemba alikuwa na niya tangu mwanzo ya

Siri li Asrari, kuweka darasa kuhusu hirizi ya majina ya Mwenyezi Mungu. Darasa hii Utendi, ameigawanya kama namna taasisi za kielimu zinavopendekeza ienreshwe

Darasa, kiskuli na ameyatowa mawazo yake kitaalamu. Nadhariya ya Usemezano Hirizi, kupitiya dhana ya Unrimi imetusaidiya kuuwangaziya utendi huu na Islam. tumefaidika na vigezo va: maudhui, uteuzi wa misamiati na mitinro ya uwasilishaji, na muktadha wa mat ${ }^{\mathrm{h}}$ umizi ya maneno hayo kama njiya ya kutambuwa aina ya lugha inayoth ${ }^{\mathrm{h}}$ umika. Kwa kwangaziya mitinro ya uwasilishaji katika darasa ya hirizi tumepata kujuwa kwamba $\mathrm{mt}^{\mathrm{t}} \mathrm{unzi}$ alikuwa na dhamira ya kutufunza badala ya kutamba hadithi ya kingano.

\section{APA CITATION}

Karama, M., Chimerah, R. \& Kineene, M. (2021). Si Tendi zothe za Kabla ya Karne ya Ishirini ni Utambaji wa Kingano Nyengine ni Ufunzaji: Kiyelelezo cha Uttendi wa Siri li Asrari. East African Journal of Swahili Studies, 3(1), 51-57. https://doi.org/10.37284/eajss.3.1.349.

\section{CHICAGO CITATION}

Karama, Mohamed, Rocha Chimerah and Mutiso Kineene. 2021. "Si Tendi zothe za Kabla ya Karne ya Ishirini ni Utambaji wa Kingano Nyengine ni Ufunzaji: Kiyelelezo cha Utendi wa Siri li Asrari”. East African Journal of Swahili Studies 3 (1), 51-57. https://doi.org/10.37284/eajss.3.1.349.

\section{HARVARD CITATION}

Karama, M., Chimerah, R. and Kineene, M. (2021) "Si Tendi zotne za Kabla ya Karne ya Ishirini ni Utambaji wa Kingano Nyengine ni Ufunzaji: Kiyelelezo cha Utendi wa Siri li Asrari”, East African Journal of Swahili Studies, 3(1), pp. 51-57. doi: 10.37284/eajss.3.1.349.

\section{IEEE CITATION}

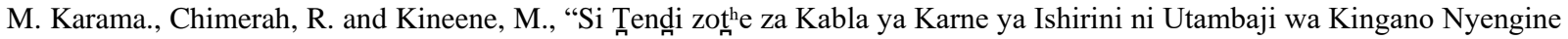
ni Ufunzaji: Kiyelelezo cha Uțendi wa Siri li Asrari”, EAJSS, vol. 3, no. 1, pp. 51-57, June. 2021.

51 | This work is licensed under a Creative Commons Attribution 4.0 International License. 


\section{MLA CITATION}

Karama, Mohamed, Rocha Chimerah and Mutiso Kineene. "Si Tendi zotn ${ }^{\text {the }}$ za Kabla ya Karne ya Ishirini ni Utambaji wa Kingano Nyengine ni Ufunzaji: Kiyelelezo cha Uțendi wa Siri li Asrari”. East African Journal of Swahili Studies, Vol. 3, no. 1, June. 2021, pp. 51-57 doi:10.37284/eajss.3.1.349.

\section{UTANGULIZI}

Utendi wa Siri li Asrari (soma Siri) una beti tisaini na zaidi zenye kuzungumziya hirizi ni nini na umuhimu wake katika maisha haya na ya akhera. Mwanamwarabu binti Bwanalemba ${ }^{1}$ (ajulikana piya kama Mwanalemba), mth unzi mwanamke wa utendi huu, anamsemesha mhusika wake, Malaika Jibril, katika beti hizo kwa njiya isiyo ya kimasimulizi ya kingano yenye kueleza 'hadithi' au 'hekaya' ili wasikilizaji wasikize na kufurahiya

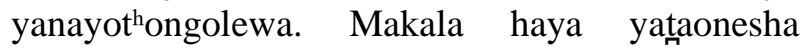
kwamba Siri ni darasa yenye muunro maalumu wa kiuwasilishaji wa kitaalamu na una malengo makhsusi yanayotarajiwa kupatikana. Imetuthubutukiya katika mapitiyo yet ${ }^{\text {th }}$ kuhusu utendi huu kuwa suala hili la Siri ina shabaha ya kufunza halijashughulikiwa kamwe.

Vijelezi vinavyotolewa na fikira inayoenrelezwa kuhusu utendi ni kwamba, "ni uth ungo mrefu wenye kwelezeya kisa au jambo kuhusu mtu/jamii" (Shariff, 1988:94). Tendi maarufu katika fasihi ya Kiswahili Hamziya, Al Inkishafi, Mwanakupona, Rasil Ghuli, Mwana Fatuma zaeleza kuhusu mat ${ }^{\mathrm{h}}$ ukio fulani katika maisha ya $\mathrm{mt}^{\mathrm{h}} \mathrm{u}$ (aghalabu Mt ${ }^{\mathrm{h}}$ ume Muhammad) na majagina wa Kiislamu. Utendi wa Mwanakupona umejitowa katika kunri hili la visa na kujiangaza kama mawaidha (wasiya). Ijapokuwa Siri ni masimulizi ya vita baina ya Mthume Muhammad (nguli wa utendi huu) na Andharuni (nruli wa utendi huu), sehemu hii ya vita, ambayo iko mwanzo wa utendi wenyewe, ina lengo la kitaalamu na wala si kutamba kisa au hadithi kama tutakavothibitisha katika makala haya.

Mapitiyo yet ${ }^{\mathrm{h}} \mathrm{u}$ ya yaliyoandikwa kuhusu utendi huu yaonesha kwamba bado hayajashughulikiya mada hii ya lengo la kiufunzaji - kwa maana ya kufanya darasa kuhusu jambo kwa mpangiliyo wa kitaalamu au kiskuli. Maelezo kuhusu istilahi ya tendi katika fasihi ya Kiswahili inajulikana, na kushikiliwa nrivo haswa mfano Parker (1982) akinukuliwa na Shariff (1984, uk. 152), kuwa ni maudhui yenye kueleza kisa kingano. Anapozungumziya uwamilifu wa utendi Shariff (1991, uk. 45) anasema kwamba, “ utendi una umuhimu mkubwa katika kuelimisha na kufurahisha jamii." Makala haya yataonesha kwamba kwelimisha huku kwatokana na kufunza kwenye mandhari ya darasani. Njogu na Chimerah (1999) na Chimerah (1989) wanatowa kiyelelezo cha namna darasa latakikana liyenreshwe wakati wa ufunzaji kama inavyopendekezwa na Taasisi ya Kutayarisha Mtalaa wa Elimu ya Kenya (K.I.C.D) na kiyelelezo hichi nricho kinachotufahamisha kwamba kwelimisha kuna mfumo na mfumo huu una miyunro inayot ${ }^{\mathrm{h}}$ umiwa ili lengo linalotarajiwa lipatikane. Makala haya hayatofwata, mguu kwa mguu, mpangiliyo huu wa Taasisi; imetosheleza kutujuza kwamba kuna miyunro ya kufunza na $\mathrm{mt}^{\mathrm{h}}$ unzi aliit ${ }^{\mathrm{h}}$ umiya miyunro fulani ili kufunza hadhira yake - wasomaji na wasikilizaji wa utendi wake.

Bakhtin (1986) anasema kwamba lugha ina lugha nyingi nrani yake ambazo hujitokeza katika mawanda tafauti tafauti na miktadha tafauti tafauti. Lugha hii inabeba maudhui ya yatakayosemwa; mitinro ya lugha makhsusi itakayoth umiwa kuyasema mawazo hayo; na, muhimu zaidi, muktadha upi utakaosababisha mawazo hayo kusemwa kwa namna inavotarajiwa. Mambo haya matatu nriyo yanayoleta athari ya kuyasema maneno hayo. Kutokana na msingi wa nadharia hii ya Usemezano inayoshadidia kuwako kwa nrimi katika lugha moja basi makala haya yatrashika aina moja ya nrimi zilizoratibishwa nayo ni: usemaji wa kitaalamu. Bakhtin (1986) anasema kila ulimi (lugha) una misamiati na mtinro tafauti wa uwasilishaji inayoitambulisha kuwa ni sajili kamili tafauti na sajili nyengine. Kwa mintarafu hii ya tafauti za kimitinro, makala haya yataangazia namna lugha hii ya kitaalamu inavojitokeza na

alimtaja Bwanalemba kuwa Ahmad bin Alwy bin Muhammad Faqihil Muqaddam "Swahibul A'maim" (mwenye malemba). 
kuutafautisha Siri na tendi nyengine maarufu kiufunzaji.

\section{UFUPISHO WA SIRI}

Siri unaanza kwa kut ${ }^{\mathrm{h}}$ ongolewa hadithi ya vita baina ya $\mathrm{Mt}^{\mathrm{h}}$ ume Muhammad na Andharuni. Andharuni aliwanyanyasa wakaaji wa mji wa kwao na wanati wakamlalamikiya $\mathrm{Mt}^{\mathrm{h}}$ ume Muhammad kuwaokowa na vitimbi va Andharuni. Mthume Muhammad akaitikiya mwith ${ }^{\text {h }}$ huwo kwa kupeleka jeshi la waislamu kuonrosha udhalimu wa Andharuni. Awali, Andharuni alionesha ujabari mkuu kwa kuwapiga waislamu bila huruma kutokana na nguvu za hirizi aliyoirithi kutoka kwa mababu zake. Mthume Muhammad kusikiya hayo alikuja kuviyona vita hivi na akaomba nusura kwa Mola ili waislamu madhaifu wasiangamizwe na Andharuni. Mungu akamjulisha, kupitiya malaika Jibril, $M t^{\text {h }}$ ume Muhammad kwamba Andharuni ana hirizi na nriyo inayompa nguvu hizo za kiajabu. $\mathrm{Mt}^{\mathrm{h}} \mathrm{ume}$ Muhammad akaomba apawe yeye hirizi hiyo na alipopawa bado alienreleya kushinrwa katika vita hivi. Baadaye, Mt ${ }^{\text {h } u m e ~ M u h a m m a d ~ a k a o m b a ~ t e n a ~}$ kwa Mungu na akamshinra Andharuni. Katika sehemu ya pili, Mt ${ }^{\text {h}}$ ume Muhammad anakuja kwelezewa na Mwalimu Jibril (malaika mtukufu) faida ya hirizi hiyo ambayo ni ya majina matukufu ya Mwenyezi Mungu. Mwandishi anatusisitiziya tushikamane na hirizi hiyo ya majina ya Mungu ili itufaidi hapa duniyani na kesho akhera.

Kifani, utendi huu unaanza na kumalizikiya kama tendi nyengine maarufu za Kiswahili kwa sifa ambazo zimedokezwa na Shariff (1988) na Mazrui na Syambo (1992). Katika utangulizi kuna kumwomba Mungu na kumswaliya $\mathrm{Mt}^{\mathrm{h} u m e}$ Muhammad na jamaa zake. Halafu ni sehemu ya vita baadaye inakuja sehemu ya mafunzo ya hirizi, na hatimaye, kuna kufunga darasa pamoja na maombi kwa waislamu. Mwisho kabisa ni kutaja

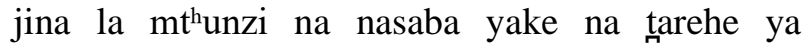
kukamilisha kuut ${ }^{\mathrm{h}}$ unga utendi huu.

\section{DARASA YA SIRI}

Tumesema huko nyuma kwamba kufunza darasani kuna mtinro wake maalumu wa uwasilishaji. Katika mtinro huu kuna miyunro-mbinu ambayo hutumiwa ili lengo linalotarajiwa lipatikane. Pili, tumesema kwamba katika kujuwa tafauti za lugha/sajili tunaweza kuangaliya misamiati inayotumiwa pamoja na mitinro ya kusema mawazo hayo. Kwa kufuwata vidokezi hivi viwili, katika sehemu hii tutaweka wazi ainați za nrimi alizot ${ }^{\mathrm{h}}{ }^{\mathrm{u}}$ miya $\mathrm{mt}^{\mathrm{th}} \mathrm{unzi}$ wa Siri kwenresha darasa yake hadi tufunukiwe kwamba Siri ilidhamiriwa kufunza na wala si utambaji wa kihadithi. Kiyelelezo cha Njogu na Chimerah (1999, uk. 141) kinaeleza kwamba darasa, kama inavyopendekezwa na K.I.E (sasa K.I.C.D), ina mpangiliyo huu: Juma (la ngapi katika mfululizo wa ufunzaji), Kipindi, Funzo, Mada, Shabaha (lengo), Somo (hatuwa mbalimbali za somo hilo), Marejeleo, Vifaa, Mazoezi, na Tathmini/Kujitathmini. Hii nriyo miyunro-mbinu ya mpangilio wa darasa. Makala haya yatachukuwa kiyelelezo hichi kama kurunzi ya kutwangaziya miyunro-mbinu hii lakini hatutotumiya mbinu zotn ${ }_{n}^{\text {the }}$ wakați wa kuuweleza Siri.

\section{Kufungua Darasa}

Siri umeanza kama tendi nyengine za kale kwa kumtaja Mungu mwanzo kabla ya kuthongowa utendi wenyewe. Baada ya kumsifu na $\mathrm{Mt}^{\mathrm{t}} \mathrm{ume}$ Muhammad na jamaa zake na kuonesha utukufu wa Muumba, Siri unaanza kwa kutowa anwani ya utendi huu. Mt ${ }^{\mathrm{h}}$ unzi anasema:

\section{Nimependa kunudhumu \\ Hadithi ya muungamu \\ Katiti iliyo tamu \\ Sikizani watambuzi.}

\section{Hadithi hinu hiyari}

\section{Isimu tawakhubiri}

Ni Siri li Asrari

Ndio itwao wayuzi.

Katika beti hizi mbili mt ${ }^{\text {h }}$ unzi anat ${ }^{\text {h }}$ wambiya jina la utrendi huu. Katika ubeti wa 22 mshororo wa tatu $\mathrm{mt}^{\mathrm{th}}$ unzi anasema utendi huu wait ${ }^{\mathrm{h}}$ wa Siri li Asrari yaani 'siri ya masiri'. Kimuunro sehemu hii imekuja baada ya sehemu ya utangulizi ambayo ililemeya katika kumuomba Mungu ampe mt ${ }^{\text {th }}$ unzi usahali wa kuthongowa utendi wake. Kwa hivyo, kwa kutupa sisi anwani ya masimulizi yake, $\mathrm{mt}^{\text {th } u n z i}$ ametupeleka mpaka darasani kutwonesha kwamba maneno yake yothe mada yake ni 'Siri li Asrari'.

Pili, katika ubeti wa 21 mshororo wa kwanza $\mathrm{mt}^{\mathrm{t}}$ unzi ametumiya neno 'nudhumu' ambalo lina maana ya kitaalamu. Hii ni istilahi ya kifasihi 
inayotafautisha 'nudhuma' na 'shairi'. Kulingana na Ustadh Mahmoud Mau (maongezi naye Novemba 2012, Lamu) pana tafauti baina ya 'nudhumu' na 'shairi'. Anasema 'nudhumu' ni "thungo zenye kumili kwenye mas'ala ya kidini na hasa yaliyot ${ }^{\text {th }}$ una na huwa hazina ubunifu (urongo). 'Shairi' ni " $t^{\text {th }}$ ungo zenye kulemea kwenye mada ambazo hazina ukali, kama mahaba na mambo mengine ya kikawaida katika jamii." Thungo za kishairi zaweza kuwa za kibunifu na zaweza kutiwa urongo nrani yake. Tukumbuke neno hili nudhumu ndilo alilot ${ }^{\text {h }}$ umiya mwenye Al Inkishafi. Kwa hivo, $m{ }^{\text {h }}$ unzi alianza utendi wake kwa kutupa anwani ya masimulizi yake na kut ${ }^{\mathrm{h}}$ wambiya kweupe kwamba ni nudhumu ambayo haina urongo ndani yake. Mas'ala ya kweleza ukweli mtupu (facts) hupatikana katika mazingira ya kitaalamu na hukusudiwa kuwapasha habari muhimu wasikilizaji. Lakini piya ni darasani ambako mwalimu hutowa mada ya somo la siku mwanzo wa darasa kama kiyelelezo cha K.I.C.D hapo juu kinavoashiriya.

\section{Usuli Na Kwenreleza Darasa}

Kama ilivo katika mapendekezo au thafiti katika tangulizi zao huwa kuna 'usuli wa utafiti'. Katika sehemu hii huelezwa nrani yake asili ya tasnifu ya mpendekezaji ilianza vipi. Kwa maneno mengine, huweza kwelezwa ilikuwaje mpaka somo hilo la utafiti likaonekana lafaa kutafitiwa. Maelezo haya ya 'usuli wa somo' tunayapata katika maelezo ya mukhtasari wa utendi ambao kwetu sisi si hadithi ya vita baina Mthume Muhammad na Andharuni (kutoka ubeti wa 32 hadi 376) bali ni maelezo ya kadimu kuhusu hirizi hii yenye nguvu za kiajabu. Yanateka muhadhara mwanzo kwa kuwapa mapisi ili waelewe maelezo muhimu yatakayokuja baadaye kuhusu hirizi. Kwet ${ }^{\mathrm{h}} \mathrm{u}$ sisi, hii ni mbinu inayopatikana katika sehemu za kitaaluma na maelezo haya yana jukumu yanayotekeleza katika kufikisha tasnifu ya msemaji. Japo $\mathrm{t}^{\mathrm{h}}$ waweza kusema ni utambaji wa hadithi ya kingano kutokana na mat ${ }^{\text {h}}$ umizi ya lugha ya kitamathali lakini kiufunzaji ina jukumu la kutowa ushahidi wa jambo lililot ${ }^{\mathrm{h}}$ ukiya au kuwapa wanafunzi asili ya wat $^{\mathrm{h}} \mathrm{u}$ kufwata au kufanya jambo fulani.

Jinsi ya kupeleka darasa tunaiyona kwanziya ubeti wa 429 - 532. Katika utendi huu, Mwalimu Jibril (mt ${ }^{\text {h}}$ unzi amemwita hivo nrani ya utendi) anamweleza kuhusu hirizi ni nini na piya kumpa faida zake. Pili, anamfundisha $\mathrm{Mt}^{\mathrm{h}} \mathrm{ume}$ Muhammad bila ya yeye $\mathrm{Mt}^{\mathrm{th}}$ ume kutiya neno hata moja katika darasa hii. Tatu, anamfunguliya $\mathrm{Mt}^{\mathrm{t}} \mathrm{ume}$ Muhammad kwamba hirizi hii ni ya majina matukufu ya Allah. Na mwishowe, anamwambiya kwamba ni jina moja tukufu la Mwenyezi Mungu nriyo liliyomo ndani ya hirizi hino.

Mtinro wa uwasilishaji wa aina hii hupatikana aghalabu katika mihadhara ya kichuwo au makongamanoni. Mhadhiri hupawa nafasi yake aelezee waliyohudhuriya maelezo yake yothe bila ya kukatizwa ili mawazo yawaingiye sawasawa waliyohudhuriya. Piya, mhadhiri nriye aliyepawa jukumu la kusema kwa kipinri hicho na wengine wothe ni wangoje hadi mwisho ikiwa wana maswali au mushkili wowothe waulize. Kwa hivyo, kwa kuwa kuna mhadhiri na kuna waliyohudhuriya taswira hii th $^{\text {h }}$ waweza kuiyona katika darasa wakati mwalimu anapowafunza wanafunzi kwa sababu yeye nriye mjuzi na wanafunzi wamet $^{\mathrm{h}}$ uliya wakimsikiza mwalimu awambiyayo.

\section{Kurejeleya Mawazo ya Wengine}

Kurejeleya mawazo ya wengine yanapot ${ }^{\mathrm{h}}$ umika katika kazi zozot ${ }_{n}^{\text {he }}$ za kitaaluma nriyo uungwana na inavotakikana kihakika. Mawazo hayo yanapawa marejeleo ili ikihitajika ithibati basi wataalamu watafwatiliya kazi zilizorejelewa na kuhakikisha; kutorejeleya mawazo ya wengine ni hatiya kubwa katika taaluma. Mt ${ }^{\text {h }}$ unzi Mwanalemba hakuwacha kurejeleya hadithi ya utendi huu aliipata wapi. Anasema:

28. Panenewa, huwambia Katika hadithi piya Nyingi zimezotukia Sahihi ya matongozi.

29. Zilio sahihi, tama Katika hadithi njema Za mtume wa kadima Nayo hiyo ni azizi.

30. Yalipokewa, yuwani Na Anasa muumini Bunu Maliki, mneni Mola mezompa razi. 
Katika ubeti wa $30 \mathrm{mt}^{\text {th }}$ unzi anasema maelezo yake yanatrokana na upokezi kutroka kwa Anas bin Malik, huyu alikuwa swahaba wa Mthume Muhammad. Katika beți za 28 na $29 \mathrm{mt}^{\mathrm{h}}$ unzi anat ${ }^{\mathrm{h}}$ wambiya kwamba hii ni Hadithi sahihi - ni ya daraja ya juu katika ukweli. Kwa hivo, anayoyasema $\mathrm{mt}^{\mathrm{h}}$ unzi yana thika ya ukweli na kwamba yanayoelezwa yanafuwata taratibu za kitaaluma ya dini kwa kutaja marejeleo ya mpokezi wa maelezo haya. Mbali ya kutaja mpokezi wa hadithi hii ni nani piya $\mathrm{mt}^{\mathrm{h}}$ unzi anafanya uwadilifu wa kitaaluma kwa kumtaja shekhe au msimulizi wa kisa hichi kila inapofika hațuwa ya kuonesha maneno hayo si yake bali ni ya $\mathrm{m}^{\mathrm{t}} \mathrm{u}$ mwengine, shekhe au mpokezi wa hadithi hii. Anarudiya usemaji huu mara kadhaa katika Siri, mfano:

\section{Kala Shekhe mtukufu Unenee msanifu Mwenye radhi ya Latifu Na Mtume muongozi.}

\section{Kala shekhe amenena Mwenye kurawi maana Hadithi ya nabiyana Muhamadi Muhijazi.}

Tutaona katika beti hizo kwamba $\mathrm{mt}^{\text {h }}$ unzi anath umiya 'kala shekhe' yenye maana ya 'amesema shekhe' na 'amenena mwenye kurawi' yenye maana ya 'amesema mwenye kuisimuliya hiyo hadithi' kurejeleya alivosema huyo $\mathrm{m}^{\mathrm{t}}$ ongowaji wa hadithi. Ni dhahiri kuwa $m \mathrm{t}^{\mathrm{h}}$ unzi mbali ya kuwa ni kazi ya kifasihi lakini alijuwa kaida za kitaalamu za kurejeleya mawazo ya wengine ili asije akakoseya adabu za kaida hizo, sembuse kuwafikiyana na mfumo wa uwasilishaji darasani wa Njogu na Chimerah (khj).

\section{Ufafanuzi Juu ya Hirizi}

Mwalimu baada ya kutowa anwani na kutowa kijelezi kuhusu mada anayoiyeleza siku hiyo huweza kutowa ufafanuzi zaidi kuhusu mada yake. Kwa mfano, ikiwa mada anayoshughulikiya ina vigawanyo katika maelezo basi atavitowa hivo vigawanyo ili wanafunzi wapate kuelewa vema mada. Azma ya mwalimu ni wanafunzi waiyelewe dhana pamoja na sehemu kadha wa kadha katika dhana hiyo. Mt ${ }^{\mathrm{h}}$ unzi Mwanalemba anatupa maana ya hirizi na piya anakuja kut ${ }^{\mathrm{h}}$ wambiya kwamba hirizi hii ina sehemu tatu ndani yake na kila sehemu ina kazi kubwa inayofanya katika kutibu maradhi. Anasema hivi:

455. Hirizi hinu Mtumi

Huzidi li aksami

Kwa mara mia msemi

Ifadhiliye wayuzi.

456. Li aksami shafi'a

Izidiye manufaa

Kwa ya sefu li kati'a

Ifadhiliye majazi.

457. Na sefu li kata'ani

Ifadhiliye yakini

Kwa maratu thalathini

Imezidi maongezi.

458. Na fadhili yenye siti

Za sefu li kati'ati

Huzidi mbingu na nti

Kwa mara kumi ndombezi.

Beți hizi zinatupa ufunuwo kwamba hirizi ina 'aksami' (ubeti wa 455 mshororo wa pili) yaani ina sehemu/vigawanyo. Kuna sehemu ya 'kati'a', 'katị'atiti', 'katâ'ani' na kila sehemu ina nguvu zake. Sehemu ya 'kati' a' (ubeti wa 456 mshororo wa tatu) ina kazi yenye kuleta pozo la magonjwa yothe. Sehemu ya 'kati'ati' (ubeti wa 458 mshororo wa pili) ina uwezo zaidi ya uzit ${ }^{\mathrm{h}} \mathrm{O}$ wa mbingu na ardhi mara kumi yake. Sehemu ya 'kata' ani' (ubeti wa 457 mshororo wa kwanza) ina nguvu zaidi ya mara thalathini. Nilipomuuliza Ustadh Mahmoud Mau (khj) kuhusu vigawanyo hivi alinambiya yaweza kuwa hirizi hii ni hiyohiyo moja lakini $\mathrm{mt}^{\mathrm{th} u n z i}$ ameipa sehemu kadhaa kwa sababu ya kutimiza arudhi ya kina katika utendi. Walakini, kwa dhahiri na maelezo ya $\mathrm{mt}^{\mathrm{h}}$ unzi mwenyewe katika utendi, tunaona kwamba kuna sehemu tatu za hirizi hii tukufu na kila moja ina kazi na uwezo wake. Kidarasa huu ni ufafanuzi wa ziyada ili wanafunzi waelewe kwa uzuri dhana ya hirizi na vigawanyo vake.

\section{Faida za Hirizi}

Katika masomo ya, kwa mfano, jiografiya ya binadamu, wanafunzi huelezwa sekta zotn $_{n}^{\text {he }}$ za kiuchumi nchini na piya huelezwa faida za sekta mbalimbali zinavojenga nchi. Mbinu hii ina ushawishi wa kuwatraka wanafunzi watiliye maanani sekta hizi na wao wajishughulishe katika 
ujenzi wa taifa kupitiya sekta hizi. Mfano huu wa kutowa dhana na halafu kutowa faida iliyo nazo kwa binadamu tunaushuhudiya katika Siri. Katika utendi huu $\mathrm{mt}^{\text {th }}$ unzi ametowa faida kumi na sita za hirizi na

402. Ni matukufu pulika

Maina yakwe Rabuka

Na mtu mwenye kwandika

Menali mangi majazi.

404. Mtu hoyo mfadhala

Kinwa maina ya Mola

Wepukane na madhila

Milele hana kinyezi.

405. Hangiwi maisha yake

Ni msiba mpulike

Katika moyoni mwake

Ila kwitwa ni Azizi.

406. Walau hamu na ghamu

Hawi nayo kwa dawamu

Mtu hoyo muadhamu

Mungu amemhifazi.

407. Na siku hiyo bashiri

Ya Kiyama kudhihiri

Hufungamana umuri

Na maina ya Muyuzi.

472. Dua hinu Mustwafa

Ni kama mtu wa sifa

Alowakifu Arafa

Siku ya Juта тиyиzi.

473.Thawabuze tatongowa

Mtu takoitukuwa

Dua hinu ya sitawa

Hapati la kumuuzi.

Katika beți hizo hapo juu, maneno tuliyoweka kwa herufi za mlazo nrizo faida za hirizi. Tunafahamishwa kwamba hirizi hii inaweza kutibu: magonjwa ya kiwiliwili (ubeti wa 404, 405, 406), magonjwa ya kiakili ya majini wabaya (ubeti wa 507), kuepusha mateso ya kaburini (ubeti wa 474, 475), mwenye hirizi hii haogopi misukosuko ya siku ya kiyama (ubeti wa 407, 477), na huko akhera $\mathrm{mt}^{\mathrm{h}} \mathrm{u}$ huyo atapawa pepo ya juu kabisa 'jannatu firdausi' (ubeti wa 479). Niya ya $\mathrm{mt}^{\text {h }}$ unzi-mwalimu ni kutaka kushawishi kwamba hirizi hii inaweza kufanya kazi ameziratibisha moja baada ya nyengine katika beti moja moja kama wafanyavo walimu wa shule madarasani. Kuhusu faidna ya hirizi $\mathrm{mt}^{\mathrm{h}}$ unzi anat ${ }^{\mathrm{h}}$ wambiy:

\section{Siku ya kufa basiri \\ Hawaonani umuri \\ Na Munkar wa Nakiri \\ Hawamwandiki maozi.}

475. Wala kabisa haoni

Zituko za kaburini

Mtu hoyo kwa yakini

Ya Rabi memhifazi.

476.Yuwa mtume rasuwa

Dua hinu ikuziwa

Ulimwenguni ni dawa

Kwa kula mawi marazi.

477. Na Ahira ni zaidi

Hutekeleza ahadi

Na kuokoka na abadi

Na harufu na kinyezi.

479. Humpa Mola Mkwasi

Pepo ya Firdausi

Kangiya ndani upesi

Ziunguni kabarizi.

507. Manufaaye basiri

Nda Rihi li Ahmari

Huziwiya kula shari

Na baa za wazimzi.

516. Na yambo ulitakalo

Lolote ulipendalo

Ukalinuwiliyalo

Hulipata uli razi.

bila wasiwasi tena anatuonesha mahali na wakati gani.

\section{Hitimisho La Darasa}

Wakatri mwalimu anapokaribiya kufunga darasa yake huweka sehemu ya hitimisho ili kutowa mukhtasari na piya kusisitiza lengo la somo hilo. Hii ni mbinu ya kimasomo ili kusakinisha mawazo ambayo yametolewa katika darasa na kumshawishi msikilizaji akubali yale aliyoelezewa baada ya kuyafahamu. Bakhtin anasema katika masemezano

56 | This work is licensed under a Creative Commons Attribution 4.0 International License. 
huwa kuna 'sentensi za mstari wa mbele' ambazo, mosi, hujulisha mipaka ya kauli za wasemezanao na, pili, hubeba msisitizo wa yale yaliyosemwa katika kauli nzima. Ni mpaka kwa sababu huonesha kauli ya $\mathrm{mt}^{\mathrm{th}} \mathrm{u}$ moja iliyanza wapi na imekomeya wapi. Sentensi hizi huwa na sifa ya kurudiliya niya yenyewe ya maneno kusemwa. Katika utendi huu kuna beti mbili ambazo ziko mbali mbali kimaweko lakini zasema kauli moja. $\mathrm{Mt}{ }^{\mathrm{h}}$ unzi anasema hivi:

\section{Tena ina manufaa \\ Mtu atakaovaa \\ Humwepukiya baa \\ Na zitimbi za gharazi.}

\section{Hirizi hinu wendaka \\ Mtu kombe kilandika \\ Anwapo na kuipaka \\ Hasaliwi ni marazi.}

Beți hizi mbili ambazo ziko mahala tafauti katika uțendi zinarejeleya jambo moja - umuhimu wa hirizi hii ya majina ya Mwenyezi Mungu. Katika ubeti wa $25 \mathrm{mt}^{\text {h }}$ unzi anat ${ }^{\text {th }}$ wambiya kwamba hirizi hii $\mathrm{mt}^{\mathrm{t}} \mathrm{u}$ atakapokuwa nayo basi hashikwi na

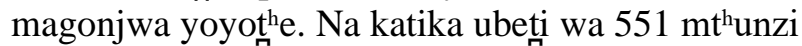
anaregeleya mawazo yayo hayo. Mwanzo wa utendi anatupa fununu kwamba hirizi hii ina manufaa makubwa na katika hitimisho lake la utendi piya anat ${ }^{\mathrm{h}}$ wambiya vivyo. Juu ya kwamba ni sentensi za mstari wa mbele lakini kwetu sisi beti hizi zaonesha hitimisho la mwalimu katika darasa yake na zasisitiza kauli yake tangu mwanzo kwamba maelezo anayoyatowa nriyo 'siri ya masiri'.

\section{HITTIMISHO}

Makala haya yamejaribu kupinruwa kauli kwamba tendi za kabla ya karne ya ishirini zilith ${ }^{\mathrm{h}}$ ungwa kweleza mapisi ya $\mathrm{mt}^{\mathrm{h}} \mathrm{u} / \mathrm{jamii}$ tu. Kutokana na miyunro-mbinu ya namna $\mathrm{mt}^{\mathrm{h}}$ unzi anavoenreleza maudhui yake imedhihirika kwamba Mwanalemba alikuwa na dhamira ya kutufunza kuhusu hirizi na umuhimu wake na aligawanya darasa yake katika vigawanyo venye kulanrana sawasawa na maelezo ya K.I.C.D. Hatukuchukuwa kiyelelezo chote cha K.I.C.D kuwa mfano wa kuenresha darasa ya mthunzi bali tumetraka kuonesha, kwa ithibați, kwamba kufunza kuna muunro unaopendekezwa na taasisi za kielimu. Kwa kufwata muunro huu, tumedhihirisha madhumuni mbalimbali aliyothumiya $m t^{\text {h }}$ unzi wa Siri ili kutufunza darasa yake juu ya hirizi: vigawanyo vake, ufaafu wake kwa magonjwa tuyaonao na tusiyoyaona, na ithibati ya kufanya kazi kwake katika hali ya kijamii kama kwenye vita baina ya $\mathrm{Mt}^{\mathrm{h}}$ ume Muhammad na Andharuni. Kutokana na tuliyobainisha ni wazi Mwanalemba alitupa darasa na alilipanga namna ya kutujuza mengi katika darasa hiyo na kwamba si kama inavofikiriwa kwamba tendi za Kiswahili za kabla ya karne ya ishirini ni utambaji wa hadithi za kingano tu. $T^{\mathrm{h}}$ waregeleya tena kuwa utendi wa Siri bado haujashughulikiwa kwa mapana na marefu yake kuna mas'ala yasiyo ya kidini kama muktadha na mandhari ya kuth ${ }^{\text {h }}$ gnga kwake yahitaji ufafanuzi ili upate kweleweka kwa uzuri zaidi.

\section{MAREJELEO}

Bakhtin, M. M. (1981). The dialogic imagination. Micheal H. (mtafsiri na mhariri) Austin: The University of Texas Press.

Bakhtin, M.M. (1986). Speech genres and other late essays. Caryl, E. \& Micheal H., (wah.) Austin: The University of Texas Press.

Chimerah, R. M. (1989). The implications of the selected works of Ngugi in theeducational thinking and practices of Kenya. Tasnifu ya Uzamifu (Haijachapishwa). Ohio University, Ohio.

Dammann, P. E. (1940). Dichtungen in der Lamu mundart des Suaheli Band 28.Hamburg: Freiderichesen.

Mazrui. A. \& Syambo, B. K. (1992). Uchambuzi wa fasihi. Nairobi: East AfricanEducational Publishers.

Njogu, K. \& Chimerah, R. (1999). Ufundishaji wa fasihi: Nadharia na mbinu. Nairobi: The Jomo Kenyatta Foundation.

Shariff, I. N. (1984). Letters to the Editor. Katika Research in African Literatures, 15 (1), 150-156. Imesomwa https://www.jstor.org/stable/3819755 .

Shariff, I. N. (1988). Tungo zetu: Msingi wa mashairi na tungo nyenginezo. New Jersey: The Red Sea Press. 